\title{
Adipokines, C-Reactive Protein and Lipid Profile Levels in Hypertensive Type 2 Diabetic Portuguese Patients
}

\author{
Maria João Neuparth ${ }^{1,2}$, Jorge Brandão Proença ${ }^{3}$, Alice Santos-Silva ${ }^{4}$ \\ and Susana Coimbra ${ }^{2,5}$ \\ ${ }^{1}$ Centro de Investigação em Actividade Física, Saúde e Lazer (CIAFEL), Universidade do Porto, Porto, \\ Portugal \\ ${ }^{2}$ CESPU, Instituto de Investigação e Formação Avançada em Ciências e Tecnologias da Saúde, \\ Gandra-PRD, Portugal \\ ${ }^{3}$ CESPU, Instituto Superior de Ciências da Saúde Norte (ISCS-N), Gandra-PRD, Portugal \\ ${ }^{4}$ Laboratório de Bioquímica, Departamento de Ciências Biológicas, Faculdade de Farmácia (FFUP), \\ Universidade do Porto, Porto, Portugal \\ ${ }^{5}$ Instituto de Biologia Molecular e Celular (IBMC), Universidade do Porto, Porto, Portugal \\ Correspondence should be addressed to: Susana Coimbra; ssn.coimbra@gmail.com
}

Received Date: 31 October 2013; Accepted Date: 8 January 2014; Published Date: 28 February 2014

Academic Editor: George S. Latsios

Copyright (c) 2014 Maria João Neuparth, Jorge Brandão Proença, Alice Santos-Silva and Susana Coimbra. Distributed under Creative Commons CC-BY 3.0

\begin{abstract}
Type 2 diabetes mellitus (T2DM) and hypertension are known to be associated. We aimed to study how hypertension may influence the levels of adipogenesis, inflammation and lipid metabolism in patients with T2DM.

We studied 57 normotensive and 23 hypertensive patients with T2DM. The circulating levels of some adipokines - adiponectin, leptin and chemerin-, C-reactive protein (CRP) and the lipid profile were evaluated.

The hypertensive group (HG) of patients with T2DM presented significantly higher weight and BMI values $\left(28.6 \pm 2.8\right.$ vs $\left.26.4 \pm 4.2 \mathrm{~kg} / \mathrm{m}^{2}\right)$ than the normotensive group of patients (NG) with T2DM. The HG, compared to the NG, presented significantly higher levels of chemerin (185 [163-218] vs 166 [125-188] ng/mL), CRP (2.40 [1.87-5.12] vs 1.78 [0.64-3.71] mg/L) and triglycerides (139 [99-192] vs 99 [76-138] mg/dL), a trend towards lower levels of adiponectin, as well as higher values of total cholesterol and increased levels of leptin more evident in males. When statistical adjustment for the confounding factor BMI was performed, the significances observed were lost.
\end{abstract}

Cite this Article as: Maria João Neuparth, Jorge Brandão Proença, Alice Santos-Silva and Susana Coimbra (2014), "Adipokines, C-Reactive Protein and Lipid Profile Levels in Hypertensive Type 2 Diabetic Portuguese Patients," Research in Endocrinology, Vol. 2014 (2014), Article ID 793235, DOI: $10.5171 / 2014.793235$ 
In summary, hypertensive patients with T2DM presented raised BMI with altered adipokine secretion, namely raised chemerin levels, abnormal lipid profile and enhanced CRP concentrations, which probably underlie the association of hypertension with obesity.

Keywords: Cardiovascular disease; adipokines; body mass index; inflammation.

\section{Introduction}

The worldwide prevalence of type 2 diabetes mellitus (T2DM) is increasing rapidly (Wild et al., 2004) and hypertension is extremely common in these patients. Indeed, approximately 70\% of patients with diabetes have hypertension (Klein et al., 1996). Diabetes and hypertension account, therefore, for a significant impact on clinical care, quality of life and public health.

Diabetes mellitus and hypertension are both risk factors for cardiovascular diseases (CVD) (Adler et al., 2000). Moreover, T2DM, CVD events and hypertension are known to be associated with obesity (Basterra-Gortari et al., 2007, Aneja et al., 2004), another known CVD risk factor.

The adipose tissue, a metabolically active organ, produces several adipokines, known to be important in the inflammatory and atherosclerotic processes (Rondinone, 2006). Adiponectin, an adipose tissuespecific adipokine, is known to present anti-inflammatory activity, protect against metabolic and cardiovascular diseases, and to play an important role in lipid metabolism and atherogenesis (Tilg and Moschen, 2008). Reduced levels of adiponectin have been reported in obesity and diabetes (Tanaka et al., 2008). Moreover,

decreased adiponectin values were reported to be associated with an increased risk for incident type 2 diabetes mellitus (Marques-Vidal et al., 2012). Reduced activity of leptin, another adipokine adipose tissue-specific, leads to severe insulin resistance and vascular dysfunction (Zhang et al., 1994). Serum leptin levels seem to reflect the body fat mass and increased plasma leptin levels were associated with T2DM, obesity, hypertension, dyslipidemia and metabolic syndrome (Sari et al., 2010).
Chemerin, also produced by adipocytes, is a recently discovered adipokine known to be associated with inflammation, adipogenesis, glucose and lipid metabolisms (Bozaoglu et al., 2007, Zabel et al., 2005, Goralski et al., 2007). Raised chemerin levels have been found in obesity, pre-diabetic states and lean, overweight and obese patients with diabetes (Bozaoglu et al., 2007, Tonjes et al., 2010, Neuparth et al., 2013). The levels of these 3 adipokines are known to be altered in obesity and T2DM, and all seem to contribute to CVD events.

Obesity, thus, higher adiposity, is characterized by a low-grade chronic inflammation, with increased levels of proinflammatory cytokines and inflammatory markers, such as C-reactive protein (CRP). The levels of CRP are positively related to body mass index (BMI) (Panagiotakos et al., 2005). Moreover, inflammation has been associated with clinical progression of atherosclerotic disease and patients with high levels of CRP seem to exhibit an increased risk for adverse cardiovascular outcome (Celik et al., 2009).

Our aim was to study how hypertension may influence the levels of adipogenesis, inflammation and lipid metabolism, in Portuguese patients with T2DM. The lipid profile, the circulating levels of CRP and of some adipokines - adiponectin, leptin and chemerin - in normotensive and hypertensive Portuguese patients with type 2 diabetes were evaluated.

\section{Materials and Methods}

\section{Subjects}

The protocol used in this study was approved by the Committee on Ethics of the Instituto Superior das Ciências da Saúde Norte, CESPU, Gandra-Paredes, Portugal. 
We performed a cross-sectional study in Portuguese adult patients with T2DM. These patients, with a previous diagnosis of T2DM, were selected from the general population and invited to participate in this study. Patients presenting inflammatory or infectious diseases, liver or kidney diseases, and receiving any kind of medication that could interfere with the study evaluations were not included in the study. An accurate and detailed interview with the patients was performed, in order to collect and record the clinical characteristics of the disease, the sociodemographic data and their habits. Patients whose statements were confusing, unclear and inconsistent were excluded from the study. Diastolic and systolic blood pressure was evaluated with a calibrated manual sphygmomanometer (mercury sphygmomanometer) and recorded after at least two consecutively concordant measures. The hypertensive patients reported home measurements of blood pressure with hypertensive values and/or were previously diagnosed as hypertensive. A group of 80 T2DM patients was enrolled in this study, after their informed consent. Two groups were established, one, the normotensive group (NG), including fifty-seven Portuguese adult T2DM patients with systolic and diastolic blood pressure lower than 140 $\mathrm{mmHg}$ and $90 \mathrm{mmHg}$, respectively, and the other, the hypertensive group (HG), including 23 adult T2DM patients with systolic and/or diastolic blood pressure equal or superior than $140 \mathrm{mmHg}$ and 90 mmHg. Sociodemographic, clinical and analytical data of the two studied groups are presented in Table 1.

Table 1 - Sociodemographic, Clinical and Analytical Data in Normotensive (NG; Defined by a Systolic and a Diastolic Blood Pressure Lower than 140 and 90 mmHg,

Respectively) and in Hypertensive (HG; Defined by a Systolic and/or a Diastolic Blood Pressure Higher than $140 \mathrm{mmHg}$ and $90 \mathrm{mmHg}$, Respectively) Portuguese Type 2 Diabetes Mellitus Patients.

\begin{tabular}{|l|c|c|c|}
\hline & $\begin{array}{c}\text { NG } \\
(\mathrm{n}=57)\end{array}$ & $\begin{array}{c}\text { HG } \\
(\mathrm{n}=23)\end{array}$ & $P$-value \\
\hline Gender (F / M) & $30 / 27$ & $10 / 13$ & 0.461 \\
\hline Age (years) & $63.1 \pm 10.5$ & $62.5 \pm 9.7$ & 0.240 \\
\hline Disease length (years) & $9.9 \pm 6.9$ & $8.0 \pm 6.2$ & 0.244 \\
\hline Age at Time of Diagnosis (years) & $53.2 \pm 10.1$ & $54.5 \pm 8.7$ & 0.583 \\
\hline BMI (kg/m²) & $26.4 \pm 4.2$ & $28.6 \pm 2.8$ & 0.025 \\
\hline Weight (kg) & $70.4 \pm 11.9$ & $80.0 \pm 11.1$ & 0.001 \\
\hline Height (m) & $1.6 \pm 0.1$ & $1.7 \pm 0.1$ & 0.111 \\
\hline Glucose (mg/dL) & $129[92-164]$ & $123[109-143]$ & 0.480 \\
\hline Glycated hemoglobin (\%) & $6.70[6.30-7.60]$ & $6.80[6.25-7.90]$ & 0.778 \\
\hline Total cholesterol (mg/dL) & $186 \pm 46$ & $207 \pm 53$ & 0.083 \\
\hline HDL-cholesterol (mg/dL) & $38.7 \pm 10.7$ & $41.1 \pm 10.4$ & 0.360 \\
\hline Triglycerides (mg/dL) & $99[76-138]$ & $139[99-192]$ & $0.021^{\phi *}$ \\
\hline Chemerin (ng/mL) & $166[125-188]$ & $185[163-218]$ & $0.032^{\phi *}$ \\
\hline Adiponectin (ng/mL) & $6464[4408-8128]$ & $4888[3687-6511]$ & 0.072 \\
\hline Leptin (ng/L) & $16.2[5.3-39.6]$ & $20.8[9.3-51.1]$ & 0.230 \\
Female & $37.9[18.8-55.8]$ & $54.4[33.2-67.0]$ & 0.102 \\
Male & $5.6[2.7-11.9]$ & $12.4[4.2-20.8]$ & 0.083 \\
\hline CRP (mg/L) & $1.78[0.64-3.71]$ & $2.40[1.87-5.12]$ & $0.022^{\phi}$ \\
\hline
\end{tabular}

Loss of significance after statistical adjustment for body mass index $(\mathrm{BMI})^{\phi}$ and for weight*; F, female; M, male; HDL, high-density lipoprotein; CRP, C-reactive protein; measurements are expressed as mean \pm standard deviation or as median values (inter quartile ranges), according to Gaussian distribution of the substances. 
The diet of the T2DM patients was low in carbohydrates and fat, and all were under treatment with oral hypoglycemic drugs. The type of medication, nutrition intake, leisure activities, smoking and alcohol drinking habits were similar in both groups of T2DM patients. Besides the oral hypoglycemic therapy, none of the patients were receiving any medication that could interfere with our results (e.g. antioxidants, anti-inflammatory drugs and anti-obesity therapies).

\section{Assays}

Blood was collected by venipuncture into tubes without anticoagulant or containing EDTA, in order to obtain serum or plasma, respectively. We evaluated the plasmatic levels of chemerin, adiponectin and leptin by enzyme-immunoassays (Chemerin ELISA, Biovendor R\&DP, Heidelber, Germany; Adiponectin, R\&D Systems, Minneapolis, USA; Leptin, Mercodia, Uppsala, Sweden). The serum levels of CRP were evaluated by immunoturbidimetry (Prestige 24i CRP Ultra, P.Z. Cormay, Lublin, Poland). The lipid profile (cholesterol, triglycerides and high-density lipoprotein cholesterol (HDLc)) and glucose levels were evaluated by enzymatic colorimetric methods (Prestige, P.Z. Cormay, Lublin, Poland). To determine the levels of glycated hemoglobin we used a spectrophotometric method (Prestige 24i $\mathrm{HbA}_{1 \mathrm{c}}$, P.Z. Cormay, Lublin, Poland).

\section{Statistical Analysis}

We used the Statistical Package for Social Sciences (SPSS, version 17 for Windows, Chicago, IL, USA) for the statistical analysis. A $P$ value lower than 0.05 was considered as statistically significant. Measurements are expressed as mean \pm standard deviation, when presenting a Gaussian distribution, or as median values (interquartile ranges) for those with a nonGaussian distribution. Comparisons between groups used Student's unpaired ttest or Mann-Whitney-U test, according to Gaussian distribution of the substances. Adjustment for confounding factors (e.g. BMI, weight) used analysis of covariance (ANCOVA), after transformation of variables (if necessary); variables were linearized by logarithmic transformation, and, afterwards, checked for a Gaussian distribution. ANCOVA allows to evaluate if the significance of the differences between groups are independent of the confounding factors.

\section{Results}

Both groups of patients with T2DM were matched for gender, age, height and disease length. Glucose and glycated hemoglobin presented similar values for both groups (Table 1).

Considering gender, only leptin levels were significantly different for females and males, presenting females higher leptin levels in the two groups studied, NG and HG $(P \leq 0.001$ in both cases).

The HG of T2DM patients presented significantly higher BMI and weight values than the NG T2DM patients. These HG of T2DM patients presented also significantly higher levels of chemerin, CRP and triglycerides, as compared to the NG patients. These patients also showed, as compared to the NG, a trend towards lower levels of adiponectin and higher values of total cholesterol. Concerning leptin values, a trend towards higher levels in male patients, when compared to males in the NG, was found (Table 1).

Considering the differences observed for BMI and weight between groups, we performed ANCOVA analysis. When statistical adjustment for the confounding factor weight was performed, a loss of statistical significance for triglycerides, although almost significant $(P=0.51)$, and for chemerin $(P=0.128)$ values was found, while the differences in CRP levels remained significantly different for the two groups $(P=0.030)$. After adjustment for BMI, chemerin, triglycerides and CRP values lost statistical significance $(P=0.106$, $P=0.063$ and $P=0.083$, respectively), suggesting that the differences in these parameters between the two groups are dependent of blood pressure and also of BMI. 


\section{Discussion}

Obesity has been shown as one of the major determinants of hypertension in the general population (Kannel, 2000). Epidemiological studies have shown that there is a strong association between obesity, BMI and hypertension (Ostchega et al., 2012, Nguyen et al., 2010, Martins et al., 2003), as well as, between obesity, hypertension and diabetes (Nguyen et al., 2008, Sanchez-Castillo et al., 2005). Hyperinsulinemia, hyperleptinemia, hypercortisolemia, renal dysfunction, altered vascular structure and function, enhanced sympathetic and reninangiotensin system activity, and blunted natriuretic peptide activity are referred as some of the contributory mechanisms to the hypertension-obesity association (Aneja et al., 2004). In accordance, we observed that patients with T2DM and hypertension showed higher weight and BMI values than those without hypertension.

Patients with T2DM and hypertension, with a raised BMI, presented an altered adipokine secretion pattern, with decreased levels of adiponectin, significant increased levels of chemerin and a trend towards an increase in leptin levels that was more evident in males.

Chemerin is known to stimulate insulindependent glucose uptake concomitant with the enhanced insulin signaling in adipocytes (Takahashi et al., 2008) and appears to act as a modulator of the expression of adipocyte genes involved in glucose and lipid homeostasis (Goralski et al., 2007). Moreover, plasma chemerin levels showed a strong and independent association with key markers of metabolic syndrome, namely obesity, high-plasma triglycerides and high blood pressure (Bozaoglu et al., 2007). We reported recently that chemerin levels were raised in lean, overweight and obese T2DM patients, and that the obese patients presented significantly higher levels of chemerin, when compared to overweight and lean patients, and to controls. In the same study, the overweight patients showed significantly higher chemerin levels, as compared with lean patients and control, and lean patients revealed significantly higher chemerin levels than the control (Neuparth et al., 2013). As far as we know, only Yang et al. studied the chemerin levels in patients with T2DM and hypertension (Yang et al., 2010) and, in accordance with our data, they found that those patients showed higher BMI and enhanced values of chemerin. Our study and Yang et al. study reinforce the association of chemerin with obesity and hypertension, both markers of metabolic syndrome. Nonetheless, when statistical adjustment for the confounding factor BMI was performed, the statistical significance for chemerin levels was lost, in opposition to Yang et al. (Yang et al., 2010). According to these authors, this association chemerin - blood pressure, may be associated to the high expression of chemerin within the kidneys, a key site of blood pressure regulation. Our data suggest that, in patients with T2DM and hypertension, high chemerin levels are not only associated with hypertension, but also probably related with obesity and diabetes itself. It seems that an adipocyte dysfunction occurs. Further studies are warranted to clarify the association between chemerin, hypertension and obesity, in T2DM.

Adiponectin has anti-inflammatory activity and plays an important role in the development of insulin resistance and atherosclerosis (Tilg and Moschen, 2008). Leptin enhances the secretion of several cytokines and a reduction in its activity leads to insulin resistance (Zhang et al., 1994, Santos-Alvarez et al., 1999). Reduced levels of adiponectin and enhanced values of leptin have been reported in obesity and in diabetes (Tanaka et al., 2008, Sari et al., 2010). Concerning adiponectin, our data is in accordance with others (Adamczak et al., 2003, Iwashima et al., 2004, Khabour et al., 2013) that reported significantly lower adiponectin levels in hypertensive T2DM patients. Moreover, drug treatment of hypertension has been associated with a decrease in blood pressure and an increase in adiponectin levels (Wago et al., 2010, Lee et al., 2010). Obesity and hypertension in T2DM have been associated with raised leptin levels (Sari et al., 2010). In 
accordance with our data, Zietz et al. found higher leptin levels in hypertensive male patients with T2DM (Zietz et al., 2000), and the authors suggested that it was related to BMI and to the higher levels of insulin. In our study no significant differences in BMI were found between females and males hypertensive patients with T2DM.

Hypertensive T2DM patients showed raised levels of triglycerides and a trend towards higher total cholesterol levels. Hypertension is often associated with dyslipidemia, and both conditions increase the risk for CVD events (Kannel, 2000). Dyslipidemia causes endothelial damage and loss of physiological vasomotor activity, which may lead to an increase in systemic blood pressure. These patients also showed higher levels of CRP, another risk factor of CVD. Increased CRP levels have been also associated with raised blood pressure (Dauphinot et al., 2009, Davey Smith et al., 2005) and with insulin resistance (Xu et al., 2008). However, after adjustment for BMI, the statistical significance for triglycerides and CRP was lost, suggesting that these levels in hypertensive T2DM patients might result from an association between hypertension and obesity.

Patients with T2DM, hypertension and excess of weight presented concomitantly other risk factors of CVD events, namely raised CRP levels, dyslipidemia and altered adipokine secretion. Thus, the need to implement medical approaches to reduce weight and blood pressure in type 2 diabetes seems crucial to diminish the risk of CVD events.

As referred, there are few studies addressing the role of chemerin in patients with T2DM, hypertension and obesity. Moreover, it is known that the distribution of adipose tissue may differ between ethnic groups. Our study, performed in a Caucasian population, and the study from Yang et al. (Yang et al., 2010), in a Chinese population, showed that chemerin levels are higher in these patients, suggesting a potential link between insulin resistance, obesity, and metabolic syndrome. These studies involve relatively small groups and, therefore, further studies in larger populations are unwarranted, to clarify the role of chemerin.

In summary, hypertensive patients with T2DM presented raised BMI with altered adipokine secretion, namely raised chemerin levels, abnormal lipid profile and enhanced CRP concentrations, which probably underlie the association of hypertension with obesity. Further studies, concerning the relationship between hypertension in T2DM and adipogenesis, lipid profile and inflammation, are warranted.

\section{Acknowledgements}

This study was supported by Centro de Investigação das Tecnologias da Saúde CITS (06-2011-CITS/CESPU).

\section{References}

Adamczak, M., Wiecek, A., Funahashi, T., Chudek, J., Kokot, F. \& Matsuzawa, Y. (2003). "Decreased Plasma Adiponectin Concentration in Patients with Essential Hypertension," American Journal of Hypertension, 16 (1) 72-5.

Adler, A. I., Stratton, I. M., Neil, H. A., Yudkin, J. S., Matthews, D. R., Cull, C. A., Wright, A. D., Turner, R. C. \& Holman, R. R. (2000). "Association of Systolic Blood Pressure with Macrovascular and Microvascular Complications of Type 2 Diabetes (UKPDS 36): Prospective Observational Study," BMJ, 321 (7258) 412-9.

Aneja, A., El-Atat, F., McFarlane, S. I. \& Sowers, J. R. (2004). "Hypertension and Obesity," Recent Progress in Hormone Research, 59 169-205.

Basterra-Gortari, F. J., Bes-Rastrollo, M., Segui-Gomez, M., Forga, L., Martinez, J. A. \& Martinez-Gonzalez, M. A. (2007). "Trends in Obesity, Diabetes Mellitus, Hypertension and Hypercholesterolemia in Spain (19972003)," Medicina Clínica (Barc), 129 (11) 405-8. 
Bozaoglu, K., Bolton, K., McMillan, J., Zimmet, P., Jowett, J., Collier, G., Walder, K. \& Segal, D. (2007). "Chemerin is a Novel Adipokine Associated with Obesity and Metabolic Syndrome," Endocrinology, 148 (10) 4687-94.

Celik, T., Iyisoy, A., Celik, M., Yuksel, U. C. \& Kardesoglu, E. (2009). "C-Reactive Protein in Chronic Heart Failure: A New Predictor of Survival," International Journal of Cardiology, 135 (3) 396-7.

Dauphinot, V., Roche, F., Kossovsky, M. P., Schott, A. M., Pichot, V., Gaspoz, J. M., Gosse, P. \& Barthelemy, J. C. (2009). "C-Reactive Protein Implications in New-Onset Hypertension in a Healthy Population Initially Aged 65 Years: The Proof Study," Journal of Hypertension, 27 (4) 736-43.

Davey Smith, G., Lawlor, D. A., Harbord, R., Timpson, N., Rumley, A., Lowe, G. D., Day, I. N. \& Ebrahim, S. (2005). "Association of CReactive Protein with Blood Pressure and Hypertension: Life Course Confounding and Mendelian Randomization Tests of Causality," Arteriosclerosis, Thrombosis, and Vascular Biology, 25 (5) 1051-6.

Goralski, K. B., McCarthy, T. C., Hanniman, E. A., Zabel, B. A., Butcher, E. C., Parlee, S. D., Muruganandan, S. \& Sinal, C. J. (2007). "Chemerin, a Novel Adipokine that Regulates Adipogenesis and Adipocyte Metabolism," J Biol Chem, 282 (38) 2817588.

Iwashima, Y., Katsuya, T., Ishikawa, K., Ouchi, N., Ohishi, M., Sugimoto, K., Fu, Y., Motone, M., Yamamoto, K., Matsuo, A., Ohashi, K., Kihara, S., Funahashi, T., Rakugi, H., Matsuzawa, Y. \& Ogihara, T. (2004). "Hypoadiponectinemia is an Independent Risk Factor for Hypertension," Hypertension, 43 (6) 1318-23.

Kannel, W. B. (2000). "Fifty Years of Framingham Study Contributions to Understanding Hypertension," Journal of Human Hypertension, 14 (2) 83-90.

Khabour, O. F., Wehaibi, S. H., Al-Azzam, S. I., Alzoubi, K. H. \& El-Akawi, Z. J. (2013). "Association of Adiponectin with
Hypertension in Type 2 Diabetic Patients: The Gender Effect," Clin Exp Hypertens, 35 (5) 361-6.

Klein, R., Klein, B. E., Lee, K. E., Cruickshanks, K. J. \& Moss, S. E. (1996). "The Incidence of Hypertension in InsulinDependent Diabetes," Archives of Internal Medicine, 156 (6) 622-7.

Lee, J.- M., Kim, J.- H., Son, H.- S., Hong, E.- G., Yu, J.- M., Han, K.- A., Min, K.- W. \& Chang, S.- A. (2010). "Valsartan Increases Circulating Adiponectin Levels without Changing HOMA-IR in Patients with Type 2 Diabetes Mellitus and Hypertension," Journal of International Medical Research, 38 (1) 234-41.

Marques-Vidal, P., Schmid, R., Bochud, M., Bastardot, F., von Kanel, R., Paccaud, F., Glaus, J., Preisig, M., Waeber, G. \& Vollenweider, P. (2012). "Adipocytokines, Hepatic and Inflammatory Biomarkers and Incidence of Type 2 Diabetes. The CoLaus Study," PLoS One, 7 (12) e51768.

Martins, D., Tareen, N., Pan, D. \& Norris, K. (2003). "The Relationship between Body Mass Index, Blood Pressure and Pulse Rate among Normotensive and Hypertensive Participants in the Third National Health and Nutrition Examination Survey (NHANES)," Cellular and Molecular Biology (Noisy-le-Grand, France) , 49 (8) 1305-9.

Neuparth, M., Proença, J., Santos-Silva, A. \& Coimbra, S. (2013). "Adipokines, Oxidized LDL and C-Reactive Protein Levels in Lean, Overweight and Obese Portuguese Patients with Type 2 Diabetes," ISRN Obesity, 2013 1-7.

Nguyen, N. T., Magno, C. P., Lane, K. T., Hinojosa, M. W. \& Lane, J. S. (2008). "Association of Hypertension, Diabetes, Dyslipidemia, and Metabolic Syndrome with Obesity: Findings from the National Health and Nutrition Examination Survey, 1999 to 2004," Journal of the American College of Surgeons, 207 (6) 928-34.

Nguyen, N. T., Nguyen, X. M., Wooldridge, J. B., Slone, J. A. \& Lane, J. S. (2010). "Association of Obesity with Risk of 
Coronary Heart Disease: Findings from the National Health and Nutrition Examination Survey, 1999-2006," Surgery for Obesity and Related Diseases, 6 (5) 465-9.

Ostchega, Y., Hughes, J. P., Terry, A., Fakhouri, T. H. \& Miller, I. (2012). "Abdominal Obesity, Body Mass Index, and Hypertension in US Adults: NHANES 20072010," Am J Hypertens, 25 (12) 1271-8.

Panagiotakos, D. B., Pitsavos, C., Yannakoulia, M., Chrysohoou, C. \& Stefanadis, C. (2005). "The Implication of Obesity and Central Fat on Markers of Chronic Inflammation: The ATTICA Study," Atherosclerosis, 183 (2) 308-15.

Rondinone, C. M. (2006). "AdipocyteDerived Hormones, Cytokines, and Mediators," Endocrine, 29 (1) 81-90.

Sanchez-Castillo, C. P., Velasquez-Monroy, O., Lara-Esqueda, A., Berber, A., Sepulveda, J., Tapia-Conyer, R. \& James, W. P. T. (2005). "Diabetes and Hypertension Increases in a Society with Abdominal Obesity: Results of the Mexican National Health Survey 2000," Public Health Nutrition, 8 (1) 53-60.

Santos-Alvarez, J., Goberna, R. \& SanchezMargalet, V. (1999). "Human Leptin Stimulates Proliferation and Activation of Human Circulating Monocytes," Cellular Immunology, 194 (1) 6-11.

Sari, R., Balci, M. K. \& Apaydin, C. (2010). "The Relationship between Plasma Leptin Levels and Chronic Complication in Patients with Type 2 Diabetes Mellitus," Metabolic Syndrome and Related Disorders, 8 (6) 499-503.z

Takahashi, M., Takahashi, Y., Takahashi, K., Zolotaryov, F. N., Hong, K. S., Kitazawa, R., Iida, K., Okimura, Y., Kaji, H., Kitazawa, S., Kasuga, M. \& Chihara, K. (2008). "Chemerin Enhances Insulin Signaling and Potentiates Insulin-Stimulated Glucose Uptake in 3T3L1 Adipocytes," FEBS Letters, 582 (5) 5738.

Tanaka, T., Tsutamoto, T., Nishiyama, K., Sakai, H., Fujii, M., Yamamoto, T. \& Horie, M. (2008). "Impact of Oxidative Stress on
Plasma Adiponectin in Patients with Chronic Heart Failure," Circulation Journal, 72 (4) 563-8.

Tilg, H. \& Moschen, A. R. (2008). "Role of Adiponectin and PBEF/Visfatin as Regulators of Inflammation: Involvement in Obesity-Associated Diseases," Clinical Science (Lond), 114 (4) 275-88.

Tonjes, A., Fasshauer, M., Kratzsch, J., Stumvoll, M. \& Bluher, M. (2010). "Adipokine Pattern in Subjects with Impaired Fasting Glucose and Impaired Glucose Tolerance in Comparison to Normal Glucose Tolerance and Diabetes," PLoS One, 5 (11) e13911.

Wago, T., Yoshimoto, T., Akaza, I., Tsuchiya, K., Izumiyama, H., Doi, M. \& Hirata, Y. (2010). "Improvement of Endothelial Function in Patients with Hypertension and Type 2 Diabetes after Treatment with Telmisartan," Hypertension Research, 33 (8) 796-801.

Wild, S., Roglic, G., Green, A., Sicree, R. \& King, H. (2004). "Global Prevalence of Diabetes: Estimates for the Year 2000 and Projections for 2030," Diabetes Care, 27 (5) 1047-53.

Xu, T., Ju, Z., Tong, W., Hu, W., Liu, Y., Zhao, L. \& Zhang, Y. (2008). "Relationship of CReactive Protein with Hypertension and Interactions between Increased C-Reactive Protein and Other Risk Factors on Hypertension in Mongolian People, China," Circulation Journal, 72 (8) 1324-8.

Yang, M., Yang, G., Dong, J., Liu, Y., Zong, H., Liu, H., Boden, G. \& Li, L. (2010). "Elevated Plasma Levels of Chemerin in Newly Diagnosed Type 2 Diabetes Mellitus with Hypertension," Journal of Investigative Medicine, 58 (7) 883-6.

Zabel, B. A., Silverio, A. M. \& Butcher, E. C. (2005). "Chemokine-Like Receptor 1 Expression and Chemerin-Directed Chemotaxis Distinguish Plasmacytoid from Myeloid Dendritic Cells in Human Blood," The Journal of Immunology, 174 (1) 244-51. 
Zhang, Y., Proenca, R., Maffei, M., Barone, M., Leopold, L. \& Friedman, J. M. (1994). "Positional Cloning of the Mouse Obese Gene and its Human Homologue," Nature, 372 (6505) 425-32.

Zietz, B., Schaffler, A., Buttner, R., Scholmerich, J. \& Palitzsch, K. D. (2000). "Elevated Levels of Leptin and Insulin but Not of TNF Alpha are Associated with Hypertension in Type 2 Diabetic Males," Experimental and Clinical Endocrinology \& Diabetes, 108 (4) 259-64. 